\title{
The Future of Digital Health
}

\author{
leuan Clay \\ Evidation Health Inc., San Mateo, CA, USA
}

Hello and welcome to this special issue of Karger Digital Biomarkers: "The Future of Digital Health."

We believe that digital measures, based on person-generated health data, are foundational to a healthier future in which care is proactive, personalized, and convenient. The current pandemic has made us all aware of the importance of virtual healthcare, whether identifying early signs of risk, providing insights for patient activation, guiding intervention, or judging efficacy and long-term benefit, digital measures provide us tools to deliver personalized care at the right time, breaking down access barriers in the process. We have a huge opportunity to consolidate recent progress into meaningful transformation of the healthcare industry.

The special issue brings together invited contributions from leaders in the field and focuses on three areas critical to building foundations for healthcare which is proactive, personalized, and convenient: further maturation of our existing capabilities, continued expansion of new capabilities and investment into the next generation of researchers who will carry the field forward.

\section{Maturation of the Field}

Updates and articles on how current capabilities are gaining regulatory and payor acceptance, further refining deployment and perspectives on next steps and challenges: (1) Patient centricity in developing new measures and developing trial protocols. (2) Updates from the IMI MOBILISE-D consortium who are seeking qualification of real-world gait parameters. (3) Progress made on pre-competitive regulatory efforts to support Parkinson's disease drug development. (4) A qualitative study examining how to convey digital measure data to clinicians. (5) Practical methods to correct for variation in wear position applied to commonly captured gait parameters.

\section{Emerging Capabilities}

Original research on passive, multimodal, predictive, behavioral measures and applications. (1) Using consumer devices to model and predict recovery from orthopedic surgery, providing a basis to tailor further intervention and support. (2) A pilot study exploring how 
fatigue symptoms can be objectively and continuously monitored using multi-sensor devices and machine learning. (3) An end-to-end, technology-agnostic platform for developing minimally invasive digital measures for aging research.

Talent

Perspectives on careers and education in digital health. (1) New roles - "Digital Navigators" - being created to support patients in engaging with virtual healthcare tools. (2) Challenges and efforts underway to train and develop the future digital health workforce.

We hope you enjoy this collection.

Ieuan Clay - Special Issue Editor

\section{Acknowledgements}

The authors thank Adam Cohen for stepping in as acting editor-in-chief to review the submission for which Dr. Dorsey is a co-author.

\section{Conflict of Interest Statement}

I.C. was previously an employee of Novartis and is currently an employee of, and holds stock options in, Evidation Health. He has received payment for lecturing on Digital Health at the ETH Zurich and FHNW Muttenz. He is an Editorial Board member at Karger Digital Biomarkers and a founding member of the Digital Medicine Society.

\section{Funding Sources}

This special issue is sponsored by Evidation Health Inc. Evidation measures health in everyday life and enables anyone to participate in groundbreaking research and health programs. Built upon a foundation of user privacy and control over permissioned health data, Evidation's Achievement platform is trusted by millions of individuals - generating data with unprecedented speed, scale, and rigor. We partner with leading healthcare companies to understand health and disease outside the clinic walls. Guided by our mission to enable and empower everyone to participate in better health outcomes, Evidation is working to bring people individualized, proactive, and accessible healthcare - faster. Founded in 2012, Evidation Health is headquartered in California with additional offices around the globe. To learn more, visit evidation.com, or follow us on Twitter @evidation.

\section{Author Contributions}

I.C. defined the concept for the special issue and invited contributions. I.C. was not involved in peer review of any contributions, nor in deciding acceptance of the contributions.

\section{Karger'}

